\title{
Aberrant attentive and inattentive brain activity to auditory negative words, and its relation to persecutory delusion in patients with schizophrenia
}

This article was published in the following Dove Medical Press journal:

Neuropsychiatric Disease and Treatment

\author{
Norichika Iwashiro,' \\ Yosuke Takano,' Tatsunobu \\ Natsubori, ' Yuta Aoki,' \\ Noriaki Yahata, ${ }^{1,2}$ Wataru \\ Gonoi, ${ }^{3}$ Akira Kunimatsu, ${ }^{3}$ \\ Osamu Abe, ${ }^{3}$ Kiyoto Kasai, ' \\ Hidenori Yamasue ${ }^{4}$ \\ 'Department of Neuropsychiatry, \\ Graduate School of Medicine, The \\ University of Tokyo, Bunkyo-ku, Tokyo, \\ Japan; ${ }^{2}$ Department of Molecular \\ Imaging and Theranostics, National \\ Institute of Radiological Sciences, \\ National Institutes for Quantum and \\ Radiological Science and Technology, \\ Chiba-city, Chiba, Japan; ${ }^{3}$ Department \\ of Radiology, Graduate School of \\ Medicine, The University of Tokyo, \\ Bunkyo-ku, Japan; ${ }^{4}$ Department of \\ Psychiatry, Hamamatsu University \\ School of Medicine, Higashi-ku, \\ Hamamatsu City, Japan
}

Correspondence: Norichika Iwashiro Department of Neuropsychiatry, Graduate School of Medicine, The University of Tokyo, 7-3-I Hongo, Bunkyo-ku, Tokyo I I 3-8655, Japan

Tel +8I 358009263

Fax $+8 I 358006894$

Email iwashiro-tky@umin.ac.jp

Hidenori Yamasue

Department of Psychiatry, Hamamatsu University School of Medicine, I-20-I Handayama, Higashi-ku, Hamamatsu City 431-3192, Japan

Email yamasue@hama-med.ac.jp

\begin{abstract}
Background and purpose: Previous research has suggested that deficits in emotion recognition are involved in the pathogenesis of persecutory delusion in schizophrenia. Although disruption in auditory and language processing is crucial in the pathophysiology of schizophrenia, the neural basis for the deficits in emotion recognition of auditorily presented language stimuli and its relation to persecutory delusion have not yet been clarified.

Patients and methods: The current functional magnetic resonance imaging study used a dichotic listening task for 15 patients with schizophrenia and 23 healthy controls matched for age, sex, parental socioeconomic background, handedness, dexterous ear, and intelligence quotient. The participants completed a word recognition task on the attended side in which a word with emotionally valenced content (negative/neutral) was presented to one ear and a different neutral word was presented to the other ear. Participants selectively attended to either ear. Results: The whole brain analysis detected the aberrant neural activity in the right inferior frontal gyrus in the patients with schizophrenia compared to that in the controls $(P<0.05$, false discovery rate-corrected). Brain activity in the right pars triangularis of the inferior frontal gyrus was significantly reduced when negatively valenced words were presented to the right ear, whereas the activity of the same region was significantly enhanced when these words were presented to the left ear, irrespective of the attended ear, in the participants with schizophrenia compared to the controls. Furthermore, this diminished brain response to auditorily presented negatively valenced words significantly correlated with severe positive symptoms $(r=-0.67$, $P=0.006)$ and delusional behavior $(r=-0.62, P=0.014)$ in the patients with schizophrenia.

Conclusion: The present results indicate that the significantly impaired brain activity in response to auditorily presented negatively valenced words in the right pars triangularis of the inferior frontal gyrus is associated with the pathogenesis of positive symptoms such as persecutory delusion. Keywords: auditory perception, inferior frontal gyrus, emotion, language, functional magnetic resonance imaging, psychosis
\end{abstract}

\section{Introduction}

Individuals with schizophrenia show deficits in emotion recognition, ${ }^{1}$ which is considered a key determinant of their functional outcome. ${ }^{2,3}$ Especially, aberrant recognition of negative emotion is reportedly associated with the pathogenesis of persecutory delusion. ${ }^{4}$ Deficits have been suggested in the recognition of emotional prosody, ${ }^{5,6}$ facial expressions, ${ }^{7,8}$ and emotionally valenced words ${ }^{9}$ in schizophrenia. Deficits in language-related information processing play an important role in the 
pathophysiology of schizophrenia, ${ }^{10,11}$ particularly in the formation of positive symptoms such as delusions. ${ }^{12-14}$ Brain network dysfunction for language processing has been indicated to predict the outcome in youth at clinically high risk for schizophrenia. ${ }^{15}$ Brain network dysfunction for language processing, ${ }^{15}$ impaired brain activity for semantic retrieval, ${ }^{16}$ and impairment in the neural network for semantic tasks ${ }^{17}$ are reported to be correlated with the severity of positive symptoms. Hence, to examine emotion recognition, visually presented negatively valenced words have often been utilized as stimuli in experiments with patients with schizophrenia. ${ }^{9}$ However, abnormality in auditory information processing is crucial for the pathophysiology of schizophrenia considering the emphasis on auditory hallucinations in the diagnostic criteria $^{18,19}$ and repeatedly reported deficits associated with auditory processing. ${ }^{20,21}$ Especially, abnormal brain activity during auditory sentence perception, ${ }^{22}$ auditory working memory, ${ }^{23}$ and verbal self-monitoring ${ }^{24}$ has been indicated to correlate with positive symptoms in schizophrenia by utilizing functional magnetic resonance imaging (fMRI). Taken together, investigating the neural basis for the processing of auditorily presented language stimuli would bring novel and significant findings in the pathophysiology of persecutory delusion in schizophrenia.

General deficits in emotional recognition irrespective of specific emotional category, such as negative or positive emotions, have been implicated in patients with schizophrenia, ${ }^{25,26}$ and a dual model has been proposed to distinguish inattentive from attentive processing of emotional information. ${ }^{27-29}$ With respect to these issues, our previous study showed that behavioral abnormality was confined to auditorily presented negatively valenced words, but not to positively valenced words in both inattentive and attentive processing, and the abnormality was correlated with delusion. ${ }^{30}$

Studies investigating language-related information processing have shown dysfunctions in the inferior frontal gyrus (IFG) of participants with schizophrenia using fMRI. ${ }^{31-33}$ Several studies have suggested that there is heterogeneity in the functional ${ }^{34}$ features of the pars opercularis (PO) and pars triangularis (PT) within the IFG. Particularly, participants with schizophrenia had smaller gray matter volume in the PT rather than that in the PO, and the smaller gray matter volume in the right PT was associated with more severe positive symptoms in individuals with schizophrenia. ${ }^{12,35}$

In this study, utilizing a similar cognitive task to that in our previous study, ${ }^{30}$ we explored the brain basis of the disturbed processing for auditorily presented negatively valenced words and its relationship with persecutory delusion in patients with schizophrenia for the first time.

\section{Materials and methods}

\section{Participants}

Forty-six Japanese adults participated in this study. Of these, 23 inpatients and outpatients with schizophrenia were recruited from the Department of Neuropsychiatry, The University of Tokyo Hospital, Japan. The patients were diagnosed with schizophrenia based on the Structured Clinical Interview for DSM-IV Axis I Disorder Clinical Version. ${ }^{19}$ Psychiatric symptoms were evaluated using the Positive and Negative Syndrome Scale (PANSS) ${ }^{36}$ immediately before the current fMRI experiment. Delusional behavior scores were calculated from the subscales of the PANSS ${ }^{35,37}$ All clinical evaluations were conducted by a psychiatrist (N.I.), fully trained in maintaining reliability and consistency in diagnoses and evaluation of symptom severity, immediately before the fMRI scanning. All patients were categorized as of paranoid subtype. Twenty-three healthy participants were recruited as controls. The intelligence quotient (IQ) was evaluated using the Japanese version of the National Adult Reading Test. ${ }^{38-40}$ The dexterous ear was decided using four question items as described in our previous study. ${ }^{30}$ The patient socioeconomic status (SES) and parental SES were assessed using the Hollingshead scale, ${ }^{41}$ and the handedness was determined using the Edinburgh Inventory. ${ }^{42}$ The neuroleptic doses were calculated based on chlorpromazine equivalents. ${ }^{43}$ The controls were screened for neuropsychiatric disorders using the Structured Clinical Interview for DSM-IV Axis I Disorder Non-Patient Edition. ${ }^{18,44}$

The exclusion criteria for both groups were current or past neurological brain injury with any known cognitive consequences or loss of consciousness for $>5$ min, history of electroconvulsive therapy, the presence of auditory abnormalities (refer to our previous study), ${ }^{30}$ and substance abuse or addiction. An additional exclusion criterion for the control group was history of psychiatric disease or family history of Axis I disorders in a first-degree relative. The ethical committee of The University of Tokyo Hospital approved this study (No 397, 1350, and 2226), and this study was conducted according to the principles expressed in the Declaration of Helsinki. After a complete explanation of the study, written informed consent was obtained from every individual.

\section{MRI scanning}

A 3-Tesla MRI scanner(GE Signa HDxt, Waukesha, WI, USA) at the University of Tokyo Hospital was used. The anatomical scanning sequence was a three-dimensional Fourier-transform spoiled gradient recalled acquisition (repetition time $=6.8 \mathrm{~s}$, slice thickness $=1 \mathrm{~mm}$, in-plane resolution $=1 \times 1 \mathrm{~mm}^{2}$ ). A trained neuroradiologist (W.G.) evaluated the MRI scans to 
confirm the absence of gross abnormality. Gradient-echo echo-planar sequences were used for functional imaging (repetition time $=2 \mathrm{~s}$, echo time $=30 \mathrm{~ms}$, flip angle $=80^{\circ}$, $3 \times 3 \times 3 \mathrm{~mm}^{3}, 35$ slices, ventral-to-dorsal interleaved acquisition). One run lasted $7 \mathrm{~min} 54 \mathrm{~s}$. One examination consisted of two such runs. The first 10 functional volumes were excluded from analysis for the equilibrium of longitudinal magnetization.

\section{Experimental procedure}

We selected two categories of emotional valence (16 negative; word number 100, 160, 182, 201, 224, 236, 257, 322, $375,397,435,460,726,782,817,851 / 32$ neutral; word number 47, 78, 83, 148, 227, 434, 477, 496, 547, 548, 562, $564,642,664,681,742,752,784,825,828,829,832,836$, $854,855,863,876,893,957,1009,1020,1036)$ from the Affective Norms for English Words ${ }^{45}$ and translated them into Japanese nouns consisted of four-morae (for example, negative; Go-u-to-u, neutral; Na-i-yo-u). Although some words were adjectives in English, we adjusted them to nouns when we translated them into Japanese. For validation, 20 healthy adults (10 male/10 female) who did not participate in the current experiment rated these words in terms of their valence, arousal, and dominance on the same 9-point Likert scale as that used in the Affective Norms for English Words. ${ }^{45}$ Word frequency was quoted from a Japanese database, ${ }^{46}$ and the number of orthographic neighbors was calculated from a Japanese thesaurus. ${ }^{47}$ The current experiment used two types of word lists that significantly differed only in emotional valence (negative/neutral) (ANOVA; $P<0.01)$, but were matched in all other language features such as arousal $(P=0.17)$, dominance $(P=0.97)$, word frequency $(P=0.13)$, and number of orthographic neighbors $(P=0.12){ }^{45,48}$ All words were transformed to auditory stimuli pronounced by a synthesized voice in neutral prosody using VoiceText $^{\circledR}$ (HOYA, Tokyo, Japan). We then processed each auditory stimulus to equalize the duration of the utterances into $1,000 \mathrm{~ms}$, spoken in a naturalistic manner using Sound Forge Pro $10^{\circledR}$ (Sony Creative Software, Middleton, WI, USA). The amplitude and pitch did not significantly differ among the negative and neutral word categories $(P=0.86$ and $P=0.43$ respectively). In addition, the mean acoustic energies of the two words, which were presented simultaneously in a dichotic paradigm, were always matched using Mitsyn ${ }^{\circledR}$ (WLH, Belmont, MA, USA) and CoolEdit Pro ${ }^{\circledR}$ (Syntrillium Software Corporation, Scottsdale, AZ, USA), according to previous studies. ${ }^{49,50}$

The task design was developed based on that presented in a previous study, ${ }^{49}$ although that study used emotional prosody in contrast to the current study utilizing the emotional content of words. The participants responded by pressing a button while listening to a pair of words. To manipulate voluntary attention orthogonally to emotional words, we used a dichotic listening paradigm in which stimuli matched for mean acoustic energy and duration were presented simultaneously to both ears (negative-neutral, neutral-neutral, or neutral-negative on the right-left sides, respectively) (see also our previous study). ${ }^{30}$ The participants were initially instructed to attend either to the right ear or the left ear. After $1 \mathrm{~s}$ of speech-stimulus presentation, the participants answered whether the word heard from the attended side was the same as the word presented on the screen by pressing a button within $2 \mathrm{~s}$. Before and after each speech stimulus, a 2.5-3.5-s standby time was provided. After an instruction to switch the attention side in the middle of the session was presented for $4 \mathrm{~s}$, the participants switched their attention to the opposite side and conducted another half session (Figure 1). Each stimulus condition (negative-neutral, neutral-negative, and neutral-neutral on the right-left sides, respectively) had eight word pairs. Each word pair was used twice, and 48 trials were completed in each session. Two different sessions were completed for each participant. The order of the attention side (right/left ear) and two different sessions was counterbalanced among the participants. For each event, the time taken to answer by pressing the button after the auditory stimuli was presented and the number of correct responses was recorded automatically with E-prime 2.0 (Psychology Software Tools, Pittsburgh, PA, USA).

\section{Behavioral analysis}

For group comparisons of behavioral data in the task, we performed repeated-measures ANOVA using either the reaction time $(\mathrm{RT})$ or the correct rate $(\mathrm{CR})$ as the dependent variable, the groups (healthy control (HC), schizophrenia) as the between-subject factors, and the attention side (right, left) and the stimulus type (negative-neutral, neutral-negative, neutral-neutral) as a within-subject factor. If a significant interaction between the group and any other factor was found, follow-up analyses using ANOVA were performed. The statistical significance level was set at $P<0.05$. All statistical analyses of behavioral data were conducted using SPSS Statistics, Version 19.0 (IBM Corporation, Armonk, NY, USA).

\section{fMRI analysis}

The fMRI data were analyzed using SPM8 (The Wellcome Department of Imaging Neuroscience, London, UK) running MATLAB 2009a (MathWorks Inc., Natick, MA, USA). 


\section{Pre-scan}

$10 \mathrm{~s}$

Voice-stimulus $1 \mathrm{~s}$

First half
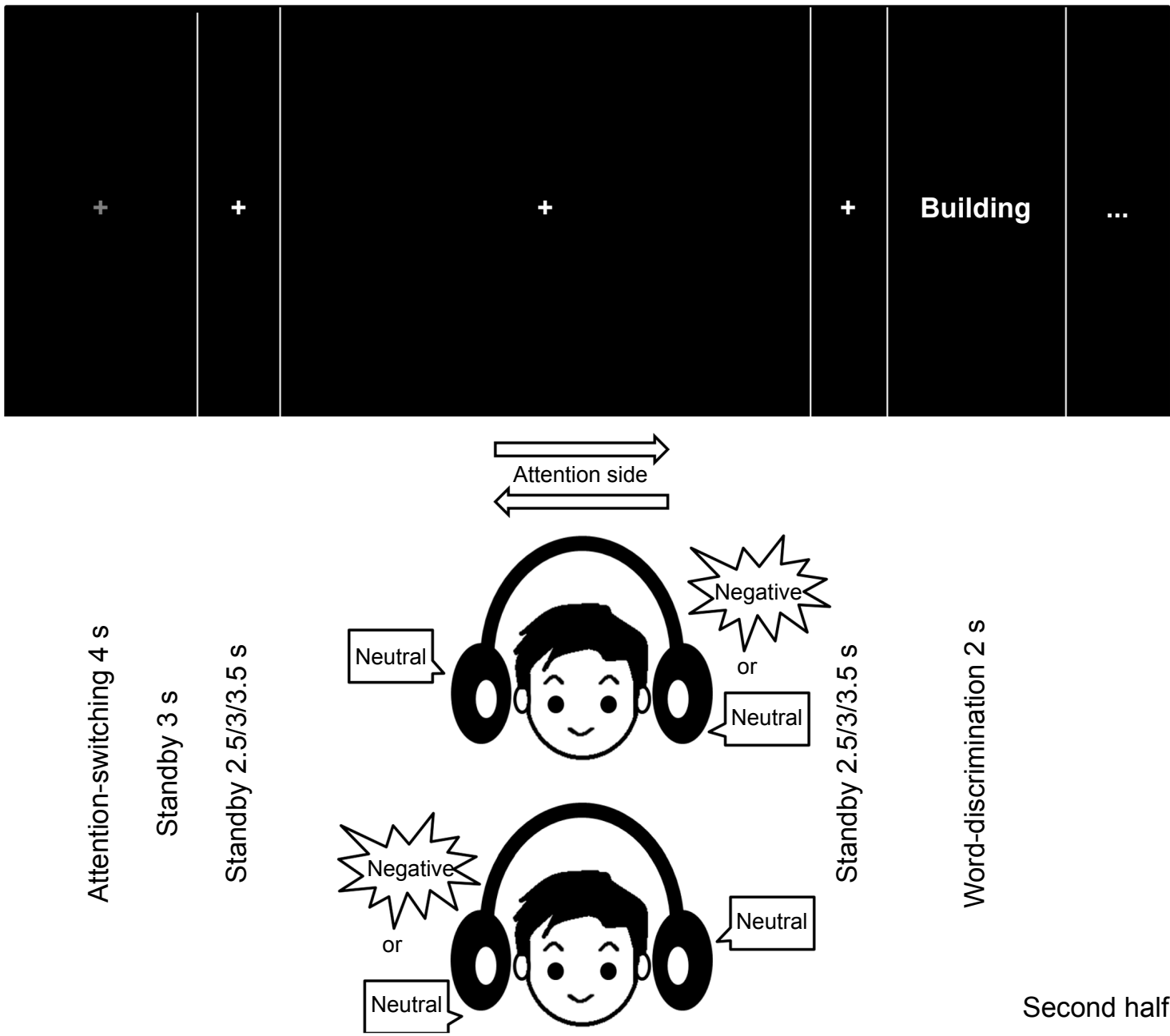

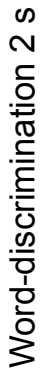
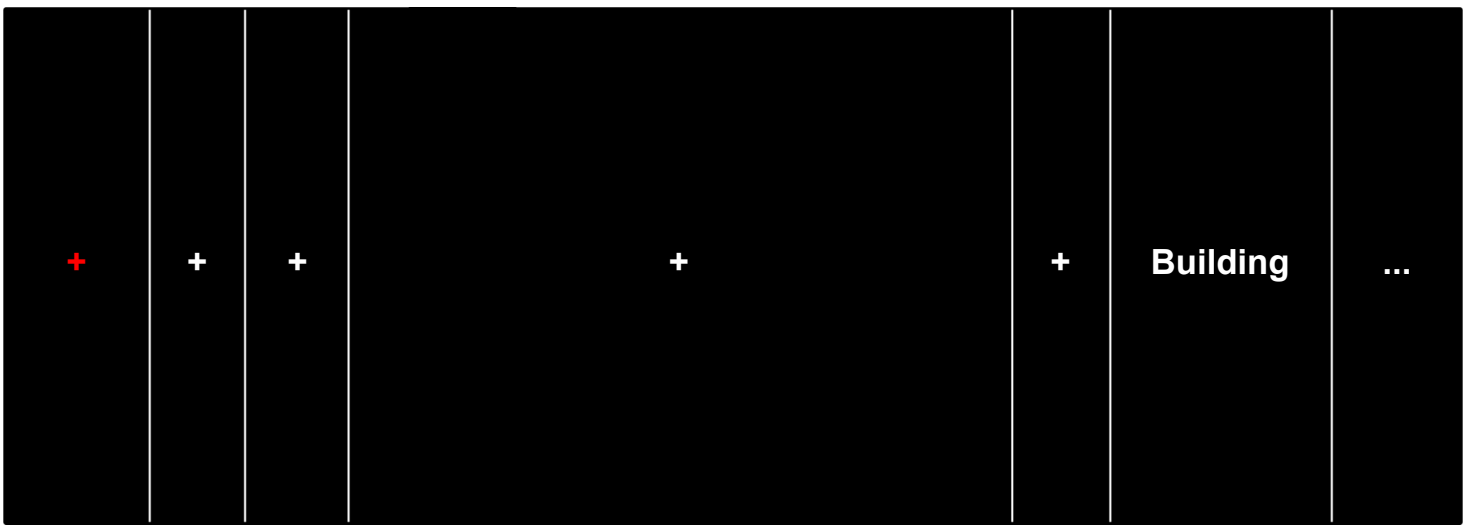

Figure I Experimental paradigm.

Notes: Different voices are presented from left and right headphones. There were three conditions ( 1 , negative word to the right ear and neutral word to the left ear; 2 , neutral word to the right ear and negative word to the left ear; 3 , neutral words to both ears). At the beginning of the task, the participants were instructed to pay attention to the right ear or to the left ear. Next, the participants answered whether the word heard from the attended ear was the same as the word consecutively presented on the screen by pressing a button. After the red cross indicating that attention should be switched to the opposite ear was presented in the middle of the session, the participants switched their attention to the other side and conducted another half session.

Functional images were realigned, slice-timing corrected, normalized to the default echo-planar imaging template, smoothed (full width at half maximum $=8 \mathrm{~mm}$, Gaussian filtered), and high-pass temporal filtered with a cutoff point of $128 \mathrm{~s}$ to remove low-frequency drift from the data. The participants included in the analysis exhibited $<3 \mathrm{~mm}$ of maximum displacement in the $\mathrm{x}, \mathrm{y}$, or $\mathrm{z}$ direction and $<3^{\circ}$ of angular rotation around each axis. ${ }^{51}$ At the single-subject 
level, we used a general linear model with three regressors (negative-neutral, neutral-negative, neutral-neutral). The six motion parameters resulting from realignment $(\mathrm{x} / \mathrm{y} / \mathrm{z}$ pitch/yaw/roll) were also included as regressors to account for residual effects of head motion. We estimated negativeneutral-minus-neutral-neutral activity and neutral-negativeminus-neutral-neutral activity when participants attended to the right ear and when they attended to the left ear, respectively (contrast images of interest), for each participant. We analyzed brain activity only for correctly answered questions. We performed a full factorial model to examine the effects of the following: group (HC, schizophrenia), contrast (negative-neutral-minus-neutral-neutral or neutral-negativeminus-neutral-neutral), attention side (right, left), and groupby-contrast, group-by-attention side, contrast-by-attention side, and group-by-contrast-by-attention side interaction. The statistical threshold was set at $P<0.05$, false discovery rate-corrected. The whole brain analysis identified brain regions where a significant interaction was found between the group and any other factor. Our task design was developed based on that of a previous study ${ }^{49}$ although that study used emotional prosody in contrast to the current study utilizing the emotional content of words. As the previous study used the signal at the peak coordinate for post-hoc tests and figures ${ }^{49}$ the blood-oxygen-level-dependent (BOLD) signal of the peak coordinate was extracted. The mean BOLD signal was defined as the "signal intensity" for the contrast of interest. Signal intensity was then used to assess the interaction.
As post-hoc analyses, we performed two-sample $t$-tests between groups. The significance level was set at $P<0.05$ for the post-hoc analysis.

\section{Correlation between signal intensity and clinical indices}

We explored the potential association between clinical symptom severity and signal intensity at the peak coordinate of significant interaction or the main group effect, by using Pearson's correlation coefficients in the schizophrenia group following the previous studies. ${ }^{37,52}$ Symptom severity included PANSS scores (positive, negative, and general psychopathology) and delusional behavior scores. Correlations between the BOLD signals and the potential confounding factors (antipsychotic doses, age, age at onset, self/parental SES, IQ, handedness) were also tested. Considering the exploratory nature of the analyses, the statistical threshold was set at $P<0.05$.

\section{Results \\ Demographic and clinical characteristics}

Five participants with schizophrenia were excluded due to excessive head motion in the fMRI scanning, and three participants with schizophrenia could not complete the fMRI task to the end. Eventually, 15 participants with schizophrenia and 23 controls were included in the statistical analysis. The control participants were matched to the participants with schizophrenia for age, sex, parental SES, ${ }^{41}$ handedness, ${ }^{42}$ dexterous ear, and estimated IQ (Table 1).

Table I Participant characteristics and symptom scores

\begin{tabular}{|c|c|c|c|c|c|c|}
\hline & \multicolumn{2}{|c|}{ Schizophrenia $(n=15)$} & \multicolumn{2}{|c|}{ Controls $(n=23)$} & \multicolumn{2}{|l|}{$t$-test } \\
\hline & Mean & SD & Mean & SD & t-value & $P$-value \\
\hline Age (range) & $29.9(18-47)$ & 8.9 & $28.5(20-4 \mathrm{I})$ & 4.3 & 0.66 & 0.52 \\
\hline Male/female & \multicolumn{2}{|l|}{$8 / 7$} & \multicolumn{2}{|l|}{$15 / 8$} & 0.54 chi-squared test & 0.46 chi-squared test \\
\hline $\mathrm{SES}^{\mathrm{a}}$ & 3.1 & 1.3 & 1.5 & 0.5 & 5.5 & $<0.01$ \\
\hline Parental SES & 2.3 & 0.6 & 2.0 & 0.6 & I.I & 0.29 \\
\hline IQ (JART25) & 109.7 & 8.9 & 112.2 & 6.4 & -1.0 & 0.33 \\
\hline Handedness ${ }^{c}$ & 91.9 & 12.5 & 89.7 & 18.8 & 0.41 & 0.69 \\
\hline Dexterous ear (right/mixed/left) & \multicolumn{2}{|l|}{$10 / 2 / 3$} & \multicolumn{2}{|l|}{$17 / 2 / 4$} & 0.29 chi-squared test & 0.87 chi-squared test \\
\hline Neuroleptic dose ${ }^{d}$ (mg/day) & 544.3 & 428.5 & & & & \\
\hline Onset of illness (years) & 23.7 & 6.7 & & & & \\
\hline Duration of illness (months) & 76.4 & 93.0 & & & & \\
\hline PANSS positive symptoms & 15.4 & 4.8 & & & & \\
\hline PANSS negative symptoms & 18.6 & 8.5 & & & & \\
\hline PANSS general psychopathology & 35.3 & 9.7 & & & & \\
\hline PANSS delusional behavior & 8.5 & 2.8 & & & & \\
\hline
\end{tabular}

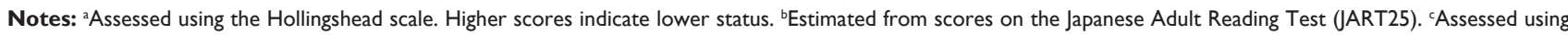
the Edinburgh Inventory ( $>0$ indicates right-handed). 'Based on chlorpromazine equivalents.

Abbreviations: IQ, intelligence quotient; PANSS, Positive and Negative Syndrome Scale; SES, socioeconomic status. 
Table 2 Behavioral results

\begin{tabular}{|c|c|c|c|c|c|}
\hline \multirow[t]{2}{*}{ Stimulus type (right ear-left ear) } & \multirow[t]{2}{*}{ Attention side } & \multicolumn{2}{|c|}{ Controls $(n=23)$} & \multicolumn{2}{|c|}{ Schizophrenia $(n=\mid 5)$} \\
\hline & & Mean & SD & Mean & SD \\
\hline \multicolumn{6}{|l|}{ Correct rate } \\
\hline \multirow[t]{2}{*}{ Negative-neutral } & Right & 0.82 & 0.07 & 0.75 & 0.11 \\
\hline & Left & 0.83 & 0.09 & 0.78 & 0.09 \\
\hline \multirow[t]{2}{*}{ Neutral-negative } & Right & 0.91 & 0.07 & 0.89 & 0.07 \\
\hline & Left & 0.90 & 0.06 & 0.85 & 0.09 \\
\hline \multirow[t]{2}{*}{ Neutral-neutral } & Right & 0.86 & 0.04 & 0.80 & 0.11 \\
\hline & Left & 0.85 & 0.09 & 0.80 & 0.10 \\
\hline \multicolumn{6}{|l|}{ Reaction time (ms) } \\
\hline \multirow[t]{2}{*}{ Negative-neutral } & Right & 947.6 & 132.6 & $1,038.6$ & 228.2 \\
\hline & Left & 931.5 & 127.6 & 980.4 & 190.9 \\
\hline \multirow[t]{2}{*}{ Neutral-negative } & Right & 911.1 & 112.0 & 923.9 & 176.3 \\
\hline & Left & 954.2 & 137.6 & 957.1 & 157.5 \\
\hline \multirow[t]{2}{*}{ Neutral-neutral } & Right & 902.8 & 135.6 & 941.5 & 195.8 \\
\hline & Left & 903.8 & 122.9 & 944.5 & 191.6 \\
\hline
\end{tabular}

In these 38 participants, the extent of head motion was not significantly different between the schizophrenia group and the control group (translation (mean/SD): schizophrenia group $0.37 \mathrm{~mm} / 0.17$, HC $0.52 \mathrm{~mm} / 0.32$, t (36) $=1.6, P=0.11$; rotation (mean $/ \mathrm{SD}$ ): schizophrenia group $0.0089^{\circ} / 0.0057$, HC $\left.0.011^{\circ} / 0.011, \mathrm{t}(36)=0.603, P=0.55\right)$.

\section{Behavioral results}

For the CR, the ANOVA showed a significant main effect of group $(F[1,36]=11.7, P=0.002)$, but no significant interaction including group (group-by-attention side: $\mathrm{F}[1,36]=0.02$, $P=0.90$; group-by-stimulus type: $\mathrm{F}[2,72]=0.57, P=0.57$; group-by-attention side-by-stimulus type: $\mathrm{F}[2,72]=0.54$, $P=0.58$ ). For RT, the ANOVA showed no significant main effect of group $(F[1,36]=0.7, P=0.40)$ or interaction including group (group-by-attention side: $\mathrm{F}[1,36]=0.4, P=0.55$; group-by-stimulus type: $\mathrm{F}[2,72]=3.0, P=0.07$; group-byattention side-by-stimulus type: $\mathrm{F}[2,72]=0.5, P=0.61$ ). These results indicated that the $\mathrm{CR}$ in the schizophrenia group was significantly worse than that in the HCs, but the RTs were not significantly different between the two groups (Table 2, Figure 2).

\section{fMRI results}

The whole brain analysis showed a significant interaction between group and contrast in the right PT of the IFG ([44 32 4], Montreal Neurological Institute coordinates, $\mathrm{k}=18, \mathrm{~F}=27.7, P<0.05$, false discovery rate-corrected). The post-hoc analyses revealed that the schizophrenia group showed significantly lower negative-neutral-minusneutral-neutral activity $(P=0.01)$ but significantly higher neutral-negative-minus-neutral-neutral activity $(P<0.01)$ than the HC group (Figure 3). No other main effect (group, attention side) or interaction (contrast-by-attention side, group-by-attention side, group-by-contrast-by-attention side) was found.

\section{Correlations between signal intensity and clinical indices}

Pearson's correlation analysis showed that the reduced negative-neutral-minus-neutral-neutral activity in the right PT of the IFG was significantly correlated with the higher PANSS positive symptom score $(r=-0.67, P=0.006)$ and delusional behavior $(r=-0.62, P=0.014)$ in the schizophrenia group (Figure 4). In contrast, there were no significant correlations between neutral-negative-minus-neutral-neutral activity and clinical symptoms $(P>0.31)$. The activity in each contrast also exhibited no significant correlation with any confounding factors $(P>0.18)$.

\section{Discussion}

To our knowledge, the present study is the first to show that brain activity in the right PT of the IFG was significantly reduced when negatively valenced words were auditorily presented to the right ear, whereas the activity was significantly enhanced when they were presented to the left ear in patients with schizophrenia compared to the control participants irrespective of being inattentively or attentively 

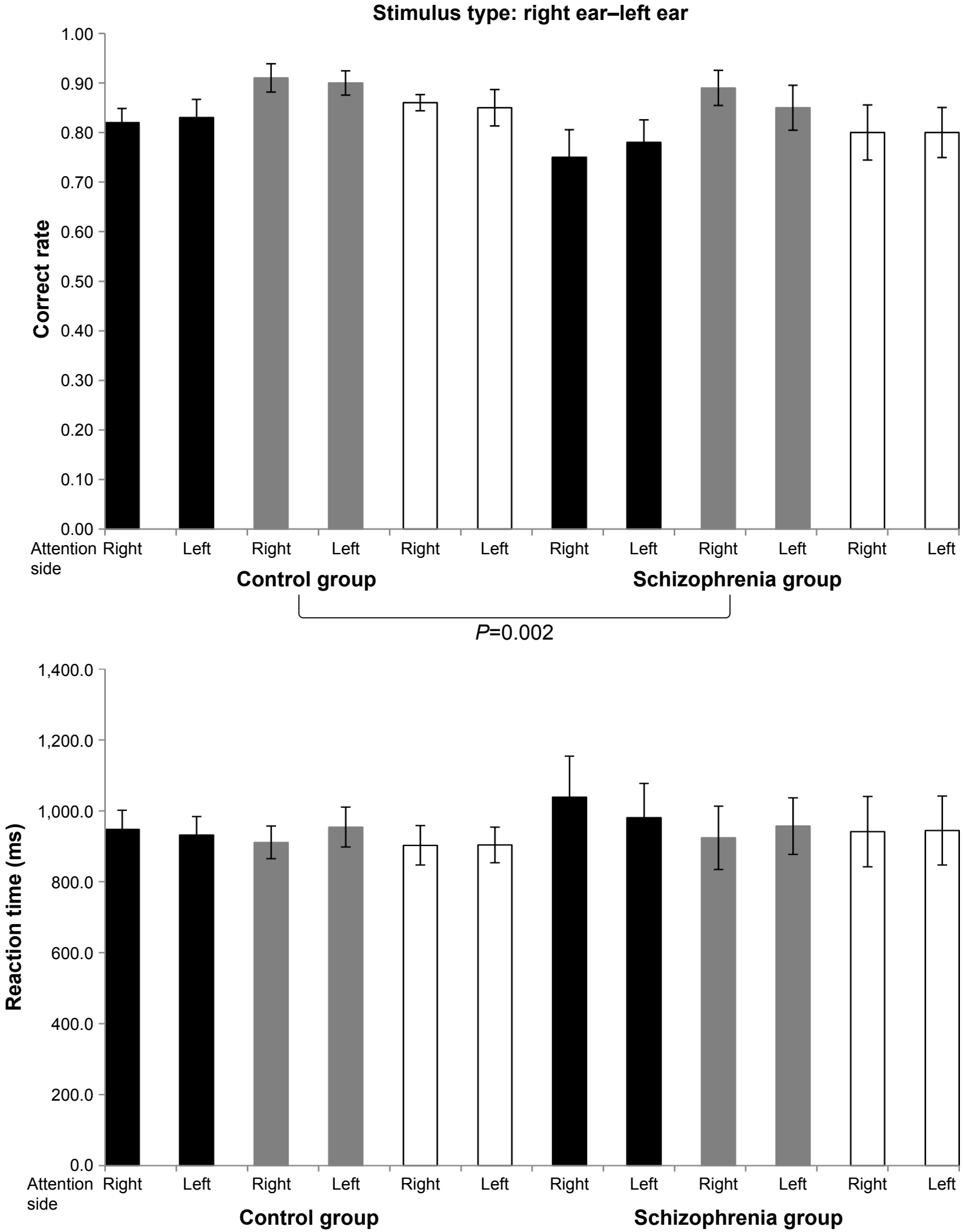

Negative-neutral

Neutral-negative Neutral-neutral

Figure 2 Behavioral results.

Notes: The correct rate in the schizophrenia group was significantly worse than that in the healthy controls $(P=0.002)$, but reaction times were not significantly different between the schizophrenia group and the control group. 

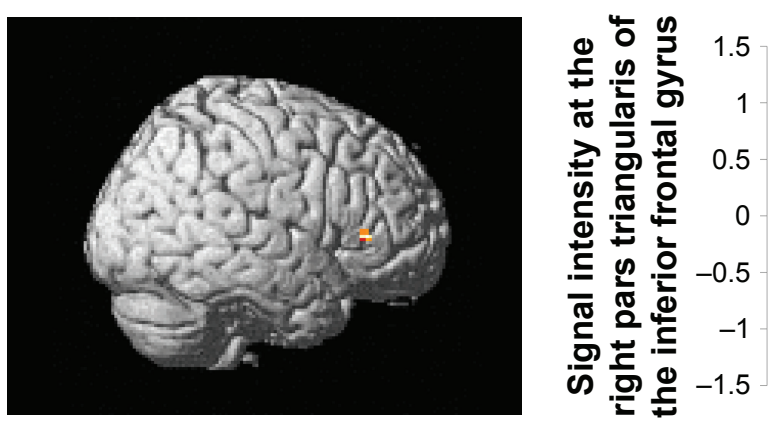

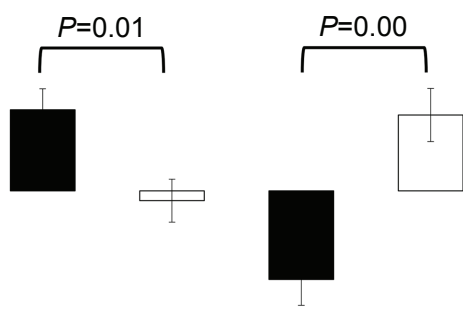

Negative-neutral Neutral-negative

Right ear-left ear

Control group

Schizophrenia group

Figure 3 Functional magnetic resonance imaging results.

Notes: Significant interaction of group and stimulus type was observed in the right pars triangularis of the inferior frontal gyrus $(P<0.05$, false discovery rate-corrected) (left image). When negative words were presented to the right ear and neutral words were presented to the left ear, activity in this region was significantly reduced in the schizophrenia group compared to that in the control group $(P=0.0 \mathrm{I})$. When neutral words were presented to the right ear and negative words were presented to the left ear, activity in this region was significantly elevated in the schizophrenia group compared to that in the control group. ( $P<0.0 \mathrm{I})($ right image).

processed. Furthermore, the decreased brain activity in the right PT when negatively valenced words were auditorily presented to the right ear significantly correlated with severe positive symptoms and delusional behavior in the patients with schizophrenia.

As we predicted in our previous study ${ }^{30}$ and based on the available literature, ${ }^{31,32,34}$ the PT of the IFG was identified as the neural basis of the disturbed processing for the auditorily presented negatively valenced words in schizophrenia. Previous studies have revealed that the PT contributes to semantic processing and the $\mathrm{PO}$ to phonological processing in the IFG. ${ }^{53,54}$ Language processes such as phonology, ${ }^{55}$ morphology, ${ }^{55}$ and syntax ${ }^{56}$ are dominant in the left hemisphere. Some language functions are mediated by the right hemisphere rather than by the left, such as discourse planning/ comprehension; understanding humor, sarcasm, metaphors, and indirect requests; and the generation/comprehension of emotional prosody.$^{10}$ Regarding the IFG, semantic processing is dominant in the left IFG, ${ }^{34,57}$ and processing of emotional content is dominant in the right IFG. ${ }^{58,59}$ Schizophrenia has been previously referred to as "a left hemisphere disorder" ${ }^{60,61}$ The higher order language functions mediated by the right hemisphere are essential for accurately understanding someone's communicative intent, and the deficits displayed by patients with schizophrenia may markedly contribute to their social interaction deficits. ${ }^{10}$ A previous study showed greater gray matter density reduction in the right IFG in UHR participants who later developed psychosis than in those

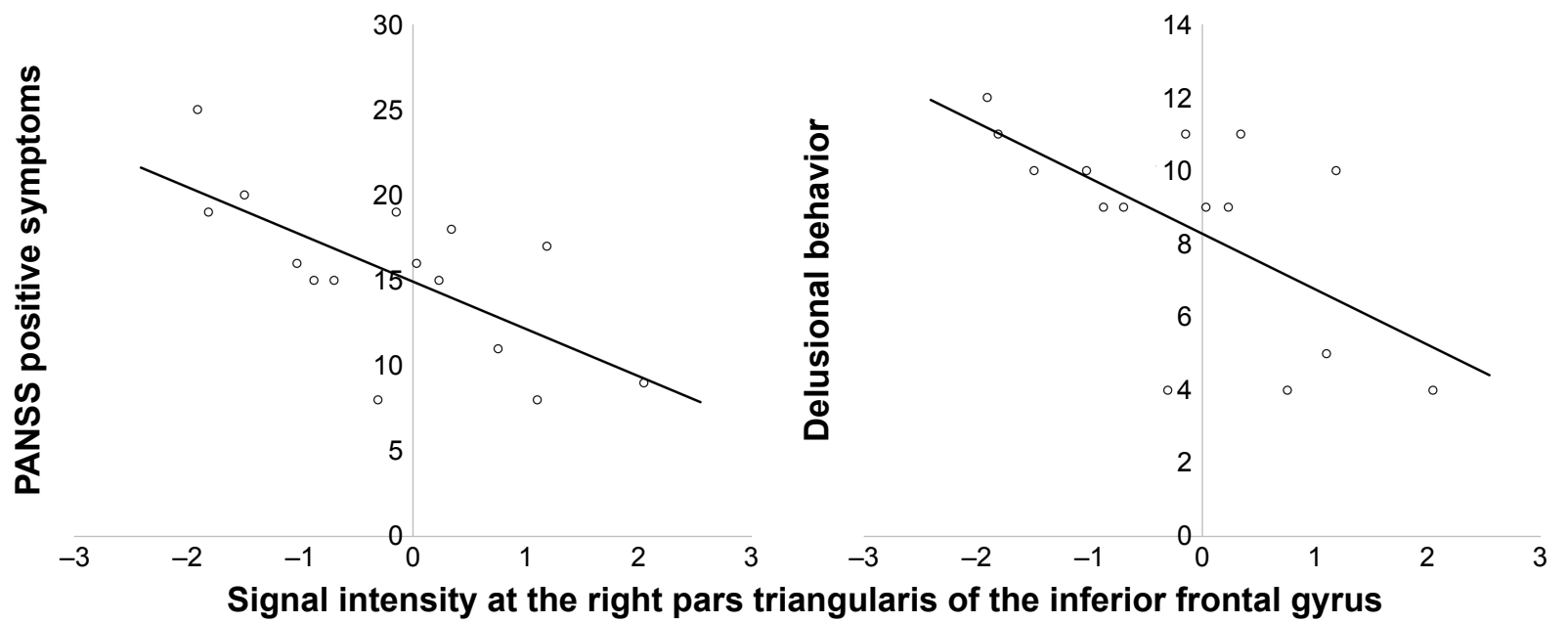

Figure 4 Relationships between signal intensity and positive symptoms and delusional behavior.

Notes: Scatter plots depict correlations between the signal intensity of negative-neutral-minus-neutral-neutral activity irrespective of attention side and severity of positive symptoms in the PANSS $(r=-0.67, P=0.006)$ and delusional behavior $(r=-0.62, P=0.014)$.

Abbreviation: PANSS, Positive and Negative Syndrome Scale. 
who did not. ${ }^{62}$ Right hemisphere network dysfunction for language processing has been proven to be correlated with positive symptoms. ${ }^{16,17}$

Most of the stimuli presented to the right ear enter contralaterally to the left hemisphere, and few stimuli enter ipsilaterally to the right hemisphere. ${ }^{63}$ A previous study utilizing magnetoencephalography indicated that emotional context was processed in the right IFG after semantic context being processed in the left IFG regarding language comprehension. ${ }^{64}$ The individuals with schizophrenia display a bilateral or reversed pattern of lateralization. ${ }^{10,65}$ The stimuli presented to the right ear entered the left IFG and might have been processed semantically and emotionally in the left IFG, and after that the rate of emotional processing in the right IFG became lower in the patients with schizophrenia than in the HCs. Therefore, brain activity in the right IFG was reduced in the patients with schizophrenia compared to that in the HCs. In the HCs, however, few stimuli entered ipsilaterally for semantic processing in the left IFG from the left ear. ${ }^{63}$ Hence, emotional processing activity was low in the right IFG after semantic processing. In the schizophrenia group, most stimuli entered from the left ear contralaterally projected to the right IFG, and might be semantically and emotionally processed simultaneously in schizophrenia because of weakness in the lateralization of brain function. ${ }^{10,65}$ Eventually, brain activity was higher in the schizophrenia group than in the control group when negatively valenced words were presented to the left ear. If semantic and emotional processing occurred simultaneously, this activity corresponded to the early phase of language processing. Hyperactivation in the right hemisphere on early-stage semantic processing has been demonstrated in schizophrenia. ${ }^{66}$ Thus, this might correspond to pre-attentive orienting bias for threat-related information in schizophrenia. ${ }^{67,68}$

The IFG has also been implicated in interpersonal interactions through imitation and observation of others' actions and emotions. ${ }^{69,70}$ A meta-analysis of fMRI studies showed that the PO reportedly performs "mirror" processing, in that it is activated during both action observation and imitation. ${ }^{70}$ Conversely, the PT does not exhibit mirror activity; it is activated only during observation. ${ }^{69}$ The activation of the PT during action observation, not during imitation, is most readily explained by the frontal inhibitory mechanisms involved in suppressing movement execution during observation or motor imagery. ${ }^{71}$ The cognitive evidence suggests the existence of a pre-attentive orienting bias for threat-related information, but also difficulty in disengaging threatening material from the conscious awareness following it; these cognitive characteristics may be associated with high levels of delusional ideation. ${ }^{67,68}$ The disruption of frontal inhibitory function in the emotional processing of auditorily presented negatively valenced words in the right PT might cause the difficulty in disengaging threatening material from conscious awareness, and therefore form positive symptoms, especially persecutory delusion.

There are several methodological limitations to our study. First, the present study sample included patients with chronic schizophrenia receiving antipsychotic medications. The effect of medication ${ }^{72}$ and chronic illness ${ }^{73}$ on our findings cannot be completely excluded, although the neuroleptic dose and duration of illness were not correlated with any neural activities. Second, the present study employing fMRI had poor temporal resolution compared to studies using magnetoencephalography. ${ }^{64,66}$ In the preceding discussion, changes in brain activity over time were described, but this is only speculation based on past data. Third, we did not execute any field-map corrections. It has been indicated that field-map corrections are not routinely performed in numerous types of fMRI studies, ${ }^{74}$ and when distortions occur along the anterior-posterior axis and the intrinsic symmetry of the brain is not affected, such benign distortion is corrected by Realign \& Unwarp in SPM8. However, EPI images at 3 Tesla can be susceptible to field inhomogeneity that may also lead to artifactual findings, and it has been indicated that field-map correction effectively improves the quality of results of task fMRI with motor tapping and auditory tasks. ${ }^{75}$ Fourth, it appeared that the neutral word list had more concrete words which have specific shape and entity, and the negative word list had more abstract words which refer to ideas or concepts. This aspect of words had not been controlled in the previous studies. ${ }^{48,76-78}$ It has been proven that abstract words produce greater activation almost exclusively in the left superior temporal and left inferior frontal cortices in contrast to concrete words. ${ }^{79-81}$ Furthermore, we recruited another 20 healthy Japanese (10 male/10 female) and asked them to rate the words used in this study for concreteness or abstractness on the same 9-point Likert scale. The ratings of concreteness or abstractness were not significantly different between the neutral words and negative words (mean/SD: negative $3.95 / 1.55$, neutral 3.27/1.95, $\mathrm{t}(46)=1.23, P=0.23$; a low score indicates concrete words). Hence, the results in this study are probably not entirely attributable to differences in the two word lists regarding concreteness and abstractness, but this influence cannot be entirely excluded. Finally, the sample size was relatively small, because the nature of the current study was exploratory. Future studies with patients with 
first-episode schizophrenia receiving minimal medication and an adequately large sample size, simultaneously employing electroencephalography and fMRI, including field-map scanning, and compiling the word list using only concrete words or abstract words could resolve these limitations.

\section{Conclusion}

Overall, taken together with our previous behavioral findings using positive, negative, and neutral auditory word stimuli, ${ }^{30}$ the present results indicate that the significantly impaired brain activity, especially for auditorily presented negatively valenced words in the right PT of the IFG, relates to the pathogenesis of persecutory delusion in patients with schizophrenia.

\section{Acknowledgments}

The authors would like to thank Chie Shimojyo and Aki Takei for their substantial support in clinical data assessment and management, and Shinsuke Koike, Yoshihiro Satomura, Mariko Tada, and Tatsuya Nagai for their substantial help with recruitment of patients. A portion of this study was also the result of a project entitled "Development of biomarker candidates for social behavior" carried out under the Strategic Research Program for Brain Sciences by the Ministry of Education, Culture, Sports, Science and Technology (MEXT).

\section{Author contributions}

N.I. contributed to project management and wrote the manuscript. Y.T., T.N., and Y.A. helped clinical evaluations and contributed to the recruitment of the participants. N.Y. gave technical support for constructing the experimental task. W.G., A.K., and O.A. supervised MRI acquisitions and evaluated all of the acquired images. K.K. and H.Y. coordinated the entire research design and took responsibility for the management of this study. All authors contributed to data analysis, drafting and revising the article, gave final approval of the version to be published, and agree to be accountable for all aspects of the work.

\section{Disclosure}

This study was supported by grants from the Ministry of Health, Labour, and Welfare (Health and Labour Science Research Grant for Comprehensive Research on Disability Health and Welfare H23-seishin-ippan-002 to H.Y. and H22-seishin-ippan-015 to K.K.) and from the JSPS/MEXT (No 22689034 to H.Y.), and a Grant-in-Aid for Scientific Research on Innovative Areas (Comprehensive Brain Science Network and Adolescent Mind \& Self-Regulation (23118001 and 23118004 ) to K.K.). The study was also supported by the National Bioscience Database Center (NBDC) of Japan Science and Technology Agency (JST) to K.K. The authors report no other conflicts of interest in this work.

\section{References}

1. Penn DL, Sanna LJ, Roberts DL. Social cognition in schizophrenia: an overview. Schizophr Bull. 2008;34(3):408-411.

2. Poole JH, Tobias FC, Vinogradov S. The functional relevance of affect recognition errors in schizophrenia. J Inter Neuropsych Soc. 2000;6(6): 649-658.

3. Kee KS, Green MF, Mintz J, Brekke JS. Is emotion processing a predictor of functional outcome in schizophrenia? Schizophr Bull. 2003; 29(3):487-497.

4. Green MJ, Phillips ML. Social threat perception and the evolution of paranoia. Neurosci Biobehav Rev. 2004;28(3):333-342.

5. Hoekert M, Kahn RS, Pijnenborg M, Aleman A. Impaired recognition and expression of emotional prosody in schizophrenia: review and meta-analysis. Schizophr Res. 2007;96(1-3):135-145.

6. Roux P, Christophe A, Passerieux C. The emotional paradox: dissociation between explicit and implicit processing of emotional prosody in schizophrenia. Neuropsychologia. 2010;48(12):3642-3649.

7. Linden SC, Jackson MC, Subramanian L, et al. Emotion-cognition interactions in schizophrenia: implicit and explicit effects of facial expression. Neuropsychologia. 2010;48(4):997-1002.

8. van 't Wout M, Aleman A, Kessels RP, Cahn W, de Haan EH, Kahn RS. Exploring the nature of facial affect processing deficits in schizophrenia. Psychiatry Res. 2007;150(3):227-235.

9. Macleod CM, Macdonald PA. Interdimensional interference in the Stroop effect: uncovering the cognitive and neural anatomy of attention. Trends Cogn Sci. 2000;4(10):383-391.

10. Mitchell RL, Crow TJ. Right hemisphere language functions and schizophrenia: the forgotten hemisphere? Brain. 2005;128(Pt 5): 963-978.

11. Delisi LE. Speech disorder in schizophrenia: review of the literature and exploration of its relation to the uniquely human capacity for language. Schizophr Bull. 2001;27(3):481-496.

12. Iwashiro N, Suga M, Takano Y, et al. Localized gray matter volume reductions in the pars triangularis of the inferior frontal gyrus in individuals at clinical high-risk for psychosis and first episode for schizophrenia. Schizophr Res. 2012;137(1-3):124-131.

13. Francis AN, Seidman LJ, Jabbar GA, et al. Alterations in brain structures underlying language function in young adults at high familial risk for schizophrenia. Schizophr Res. 2012;141(1):65-71.

14. Takahashi T, Wood SJ, Yung AR, et al. Progressive gray matter reduction of the superior temporal gyrus during transition to psychosis. Arch Gen Psychiatry. 2009;66(4):366-376.

15. Sabb FW, van Erp TG, Hardt ME, et al. Language network dysfunction as a predictor of outcome in youth at clinical high risk for psychosis. Schizophr Res. 2010;116(2-3):173-183.

16. Jamadar S, O'Neil KM, Pearlson GD, et al. Impairment in semantic retrieval is associated with symptoms in schizophrenia but not bipolar disorder. Biol Psychiatry. 2013;73(6):555-564.

17. Wu CH, Hwang TJ, Chen PJ, et al. Reduced structural integrity and functional lateralization of the dorsal language pathway correlate with hallucinations in schizophrenia: a combined diffusion spectrum imaging and functional magnetic resonance imaging study. Psychiatry Res. 2014;224(3):303-310.

18. American Psychiatric Association. Diagnostic and statistical manual of mental disorders. 4th ed. Washington, DC: American Psychiatric Press; 1994

19. First MB, Spitzer RL, Gibbon M, Williams JBW. Structured clinical interview for DSM-IV axis I disorders: clinical version (SCID-CV). Washington, DC: American Psychiatric Press; 1997.

20. Kasai K, Shenton ME, Salisbury DF, et al. Progressive decrease of left superior temporal gyrus gray matter volume in patients with firstepisode schizophrenia. Am J Psychiatry. 2003;160(1):156-164. 
21. Fisher M, HollandC,Merzenich MM, Vinogradov S. Using neuroplasticitybased auditory training to improve verbal memory in schizophrenia. Am J Psychiatry. 2009;166(7):805-811.

22. Plaze M, Bartrés-Faz D, Martinot JL, et al. Left superior temporal gyrus activation during sentence perception negatively correlates with auditory hallucination severity in schizophrenia patients. Schizophr Res. 2006;87(1-3):109-115.

23. Menon V, Anagnoson RT, Mathalon DH, Glover GH, Pfefferbaum A. Functional neuroanatomy of auditory working memory in schizophrenia: relation to positive and negative symptoms. Neuroimage. 2001;13(3): 433-446.

24. Kumari V, Fannon D, Ffytche DH, et al. Functional MRI of verbal selfmonitoring in schizophrenia: performance and illness-specific effects. Schizophr Bull. 2010;36(4):740-755.

25. Bach DR, Buxtorf K, Grandjean D, Strik WK. The influence of emotion clarity on emotional prosody identification in paranoid schizophrenia. Psychol Med. 2009;39(6):927-938.

26. Leitman DI, Hoptman MJ, Foxe JJ, et al. The neural substrates of impaired prosodic detection in schizophrenia and its sensorial antecedents. Am J Psychiatry. 2007;164(3):474-482.

27. Gorno-Tempini ML, Pradelli S, Serafini M, et al. Explicit and incidental facial expression processing: an fMRI study. Neuroimage. 2001;14(2): 465-473.

28. Winston JS, O’Doherty J, Dolan RJ. Common and distinct neural responses during direct and incidental processing of multiple facial emotions. Neuroimage. 2003;20(1):84-97.

29. Mathersul D, Palmer DM, Gur RC, et al. Explicit identification and implicit recognition of facial emotions: II. core domains and relationships with general cognition. J Clin Exp Neuropsychol. 2009;31(3): 278-291.

30. Iwashiro N, Yahata N, Kawamuro Y, Kasai K, Yamasue H. Aberrant interference of auditory negative words on attention in patients with schizophrenia. PLoS One. 2013;8(12):e83201.

31. Raij TT, Valkonen-Korhonen M, Holi M, Therman S, Lehtonen J, Hari R. Reality of auditory verbal hallucinations. Brain. 2009;132(Pt 11): 2994-3001.

32. Ragland JD, Gur RC, Valdez J, et al. Event-related fMRI of frontotemporal activity during word encoding and recognition in schizophrenia. Am J Psychiatry. 2004;161(6):1004-1015

33. Jung WH, Jang JH, Shin NY, et al. Regional brain atrophy and functional disconnection in Broca's area in individuals at ultra-high risk for psychosis and schizophrenia. PLoS One. 2012;7(12):e51975.

34. Nishitani N, Schürmann M, Amunts K, Hari R. Broca's region: from action to language. Physiology. 2005;20:60-69.

35. Suga M, Yamasue H, Abe O, et al. Reduced gray matter volume of Brodmann's area 45 is associated with severe psychotic symptoms in patients with schizophrenia. Eur Arch Psychiatry Clin Neurosci. 2010; 260(6):465-473.

36. Kay SR, Fiszbein A, Opler LA. The positive and negative syndrome scale (PANSS) for schizophrenia. Schizophr Bull. 1987;13(2): 261-276.

37. Takano Y, Aoki Y, Yahata N, et al. Neural basis for inferring false beliefs and social emotions in others among individuals with schizophrenia and those at ultra-high risk for psychosis. Psychiatry Res Neuroimaging. 2017; 259:34-41.

38. Matsuoka K, Kim Y. Japanese Adult Reading Test (JART). Tokyo: Shinkou-Igaku publishers; 2006.

39. Matsuoka K, Uno M, Kasai K, Koyama K, Kim Y. Estimation of premorbid IQ in individuals with Alzheimer's disease using Japanese ideographic script (kanji) compound words: Japanese version of national adult reading test. Psychiatry Clin Neurosci. 2006;60(3): 332-339.

40. Uetsuki M, Matsuoka K, Kim Y. Estimation of premorbid IQ by JART in schizophrenia. Seishin Igaku. 2006;48(1):15-22.

41. Hollingshead AB. Two-factor index of social position. New Haven, CT: Yale University Press; 1957.

42. Oldfield RC. The assessment and analysis of handedness: the Edinburgh inventory. Neuropsychologia. 1971;9(1):97-113.
43. Andreasen NC, Pressler M, Nopoulos P, Miller D, Ho BC. Antipsychotic dose equivalents and dose-years: a standardized method for comparing exposure to different drugs. Biol Psychiatry. 2010;67(3): 255-262.

44. First MB, Spitzer RL, Gibbon M, Williams JBW. Structured clinical interview for DSM-IV axis I disorders, non-patient ED. New York: Biometrics Research Department, New York State Psychiatric Institute; 1997.

45. Bradley MM, Lang PJ. Affective Norms for English Words (ANEW): stimuli, instruction manual and affective ratings. Technical report C-1. Gainesville, FL: The Center for Research in Psychophysiology, University of Florida; 1999.

46. Ikehara S, Miyazaki M, Shirai S. Goi-Taikei - A Japanese Lexicon CDROM. Tokyo: Iwanami Shoten; 1999.

47. Ikegami A, Kaneda H, Sugisaki K. Daijisen. Matsumura a. Tokyo: Shogakukan; 1998.

48. Gaillard R, Del Cul A, Naccache L, Vinckier F, Cohen L, Dehaene S. Nonconscious semantic processing of emotional words modulates conscious access. Proc Natl Acad Sci U S A. 2006;103(19): 7524-7529.

49. Grandjean D, Sander D, Pourtois G, et al. The voices of wrath: brain responses to angry prosody in meaningless speech. Nat Neurosci. 2005; $8(2): 145-146$.

50. Aue T, Cuny C, Sander D, Grandjean D. Peripheral responses to attended and unattended angry prosody: a dichotic listening paradigm. Psychophysiology. 2011;48(3):385-392.

51. Liu Y, Liang M, Zhou Y, et al. Disrupted small-world networks in schizophrenia. Brain. 2008;131(Pt 4):945-961.

52. Watanabe T, Yahata N, Abe O, et al. Diminished medial prefrontal activity behind autistic social judgments of incongruent information. PLoS One. 2012;7(6):e39561.

53. Costafreda SG, Fu CH, Lee L, Everitt B, Brammer MJ, David AS. A systematic review and quantitative appraisal of fMRI studies of verbal fluency: role of the left inferior frontal gyrus. Hum Brain Mapp. 2006; 27(10):799-810.

54. Saetrevik B, Specht K. Cognitive conflict and inhibition in primed dichotic listening. Brain Cogn. 2009;71(1):20-25.

55. Titone D, Levy DL. Lexical competition and spoken word identification in schizophrenia. Schizophr Res. 2004;68(1):75-85.

56. Condray R, Steinhauer SR, van Kammen DP, Kasparek A. The language system in schizophrenia: effects of capacity and linguistic structure. Schizophr Bull. 2002;28(3):475-490.

57. Bookheimer S. Functional MRI of language: new approaches to understanding the cortical organization of semantic processing. Annu Rev Neurosci. 2002;25(1):151-188.

58. Buchanan TW. Retrieval of emotional memories. Psychol Bull. 2007; 133(5):761-779.

59. Sass K, Habel U, Kellermann T, Mathiak K, Gauggel S, Kircher T. The influence of positive and negative emotional associations on semantic processing in depression: an fMRI study. Hum Brain Mapp. 2014;35(2):471-482.

60. Oh TM, Mccarthy RA, Mckenna PJ. Is there a schizophasia? A study applying the single case approach to formal thought disorder in schizophrenia. Neurocase. 2002;8(3):233-244.

61. Crow TJ. Schizophrenia as the price that Homo sapiens pays for language: a resolution of the central paradox in the origin of the species Brain Res Brain Res Rev. 2000;31(2-3):118-129.

62. Pantelis C, Velakoulis D, Mcgorry PD, et al. Neuroanatomical abnormalities before and after onset of psychosis: a cross-sectional and longitudinal MRI comparison. Lancet. 2003;361(9354): 281-288.

63. Nieuwenhuys R. Anatomy of the auditory pathways, with emphasis on the brain stem. Adv Otorhinolaryngol. 1984;34:25-38.

64. Ihara A, Wei Q, Matani A, et al. Language comprehension dependent on emotional context: a magnetoencephalography study. Neurosci Res. 2012;72(1):50-58.

65. Natsubori T, Hashimoto R, Yahata N, et al. An fMRI study of visual lexical decision in patients with schizophrenia and clinical high-risk individuals. Schizophr Res. 2014;157(1-3):218-224. 
66. Zeev-Wolf M, Faust M, Levkovitz Y, Harpaz Y, Goldstein A. Magnetoencephalographic evidence of early right hemisphere overactivation during metaphor comprehension in schizophrenia. Psychophysiology. 2015;52(6):770-781.

67. Arguedas D, Green MJ, Langdon R, Coltheart M. Selective attention to threatening faces in delusion-prone individuals. Cogn Neuropsychiatry. 2006;11(6):557-575.

68. Bentall RP, Kaney S. Content specific information processing and persecutory delusions: an investigation using the emotional Stroop test. Br J Med Psychol. 1989;62(Pt 4):355-364.

69. Iacoboni M. Neurobiology of imitation. Curr Opin Neurobiol. 2009; 19(6):661-665.

70. Molnar-Szakacs I, Iacoboni M, Koski L, Mazziotta JC. Functional segregation within pars opercularis of the inferior frontal gyrus: evidence from fMRI studies of imitation and action observation. Cereb Cortex. 2005;15(7):986-994.

71. Deiber MP, Ibañez V, Honda M, Sadato N, Raman R, Hallett M. Cerebral processes related to visuomotor imagery and generation of simple finger movements studied with positron emission tomography. Neuroimage. 1998;7(2):73-85.

72. Vernon AC, Natesan S, Modo M, Kapur S. Effect of chronic antipsychotic treatment on brain structure: a serial magnetic resonance imaging study with ex vivo and postmortem confirmation. Biol Psychiatry. 2011;69(10):936-944.

73. Kasai K, Shenton ME, Salisbury DF, et al. Progressive decrease of left Heschl gyrus and planum temporale gray matter volume in first-episode schizophrenia: a longitudinal magnetic resonance imaging study. Arch Gen Psychiatry. 2003;60(8):766-775.
74. Togo H, Rokicki J, Yoshinaga K, et al. Effects of field-map distortion correction on resting state functional connectivity MRI. Front Neurosci. 2017;11:656.

75. Cusack R, Brett M, Osswald K. An evaluation of the use of magnetic field maps to undistort echo-planar images. Neuroimage. 2003;18(1): $127-142$.

76. Rao NP, Arasappa R, Reddy NN, Venkatasubramanian G, Reddy YC. Emotional interference in obsessive-compulsive disorder: a neuropsychological study using optimized emotional Stroop test. Psychiatry Res. 2010;180(2-3):99-104.

77. Rao NP, Jayaram N, Vishwanath V, Kalmady S, Venkatasubramanian G, Gangadhar BN. Antithetical relative emotional interference in bipolar disorder and schizophrenia: an optimized emotional stroop study. Bipolar Disorders. 2011;13:80-81.

78. Demily C, Attala N, Fouldrin G, et al. The emotional Stroop task: a comparison between schizophrenic subjects and controls. Eur Psychiatry. 2010;25(2):75-79.

79. Sabsevitz DS, Medler DA, Seidenberg M, Binder JR. Modulation of the semantic system by word imageability. Neuroimage. 2005;27(1): 188-200.

80. Noppeney U, Price CJ. Retrieval of abstract semantics. Neuroimage. 2004;22(1):164-170.

81. della Rosa PA, Catricalà E, Canini M, Vigliocco G, Cappa SF. The left inferior frontal gyrus: a neural crossroads between abstract and concrete knowledge. Neuroimage. 2018;175:449-459.
Neuropsychiatric Disease and Treatment

\section{Publish your work in this journal}

Neuropsychiatric Disease and Treatment is an international, peerreviewed journal of clinical therapeutics and pharmacology focusing on concise rapid reporting of clinical or pre-clinical studies on a range of neuropsychiatric and neurological disorders. This journal is indexed on PubMed Central, the 'PsycINFO' database and CAS,

\section{Dovepress}

and is the official journal of The International Neuropsychiatric Association (INA). The manuscript management system is completely online and includes a very quick and fair peer-review system, which is all easy to use. Visit http://www.dovepress.com/testimonials.php to read real quotes from published authors. 